\title{
La RSE : nouvelle forme de dé-marchandisation du monde?
}

Une lecture institutionnaliste à partir de Karl Polanyi

CSR: a new way of de-marchanisation of the world?

\section{Nicolas Postel et Richard Sobel}

\section{(2) OpenEdition}

\section{Journals}

Édition électronique

URL : http://journals.openedition.org/developpementdurable/8506

DOI : 10.4000/developpementdurable.8506

ISSN : 1772-9971

Éditeur

Association DD\&T

Référence électronique

Nicolas Postel et Richard Sobel, «La RSE : nouvelle forme de dé-marchandisation du monde?», Développement durable et territoires [En ligne], Vol. 1, n 3 | Décembre 2010, mis en ligne le 08 janvier 2013, consulté le 30 avril 2019. URL : http://journals.openedition.org/developpementdurable/8506 DOI : 10.4000/developpementdurable.8506

Ce document a été généré automatiquement le 30 avril 2019.

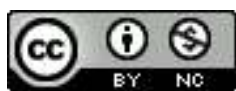

Développement Durable et Territoires est mis à disposition selon les termes de la licence Creative Commons Attribution - Pas d'Utilisation Commerciale 4.0 International. 


\title{
La RSE : nouvelle forme de dé- marchandisation du monde?
}

\author{
Une lecture institutionnaliste à partir de Karl Polanyi \\ CSR: a new way of de-marchanisation of the world?
}

\author{
Nicolas Postel et Richard Sobel
}

Ce travail a bénéficié d'une aide de l'Agence nationale de la recherche portant la référence ANR-09JCJC-0132-01

1 En matière de développement durable, les pouvoirs politiques ont déployé un arsenal de mesures, parmi lesquelles un appel aux entreprises pour que celles-ci deviennent responsables. En France, cet appel a pris la forme, en 2001, de la loi NRE ${ }^{1}$. En Europe, cet appel a pris la forme, la même année, d'une directive européenne engageant les entreprises à être responsables et à la rédaction d'un livre vert cadrant le champ de ces nouvelles responsabilités. On peut donc dater de cette année-là la reconnaissance officielle de cette forme de régulation hybride ${ }^{2}$ qu'est le mouvement de la responsabilité sociale des entreprises (désormais: RSE). Ce mouvement prend ainsi une forme clairement identifiable à partir de son repérage par le livre vert de la commission européenne de 2001 (définition réaffirmée en 2006): «la responsabilité sociale des entreprises (CSR) est un concept par lequel les compagnies intègrent des soucis sociaux et environnementaux dans leurs opérations commerciales et dans leur interaction avec leurs soustraitants sur une base volontaire. Elle concerne des entreprises décidant d'aller au-delà des conditions légales minimum et des engagements provenant des conventions collectives afin de satisfaire les besoins sociaux». (European Commission, 2006:1) ${ }^{3}$. Cette définition met l'accent sur deux points essentiels. D'une part, la RSE est qualifiée de démarche volontaire des entreprises; d'autre part, elle suppose que les engagements pris aillent au-delà de la loi. Deux points essentiels, et bien sûr, très imprécis, puisque la loi à laquelle il est fait référence, n'est pas rapportée à un territoire, et que le caractère "volontaire " de l'engagement suggère une entreprise parfaitement libre d'agir, ne subissant pas de pressions concurrentielles, actionnariales, sociétales qu'il conviendrait d'analyser. Pour le dire autrement : ce travail exploratoire de la commission européenne - un livre vert est 
une catégorie de texte non pas réglementaire mais ouvert à discussion - oublie tout le contexte dans lequel la RSE se déroule. Ce contexte est institutionnel, au sens ou ces caractéristiques relèvent de phénomènes historiques réversibles et mouvants.

Or, précisément, ce contexte institutionnel est généralement négligé par les analyses traditionnelles de la RSE menées en science de gestion. Celles-ci relèvent presque entièrement d'une approche en termes de parties prenantes, approches micro économiques et contractualistes qui ne permettent pas de cerner l'enjeu du phénomène, précisément parce qu'elles font l'impasse sur sa dimension institutionnelle (Cazal (2008), Mercier (2006), Boidin, Postel, Rousseau (2009)). Dans cet article, nous défendrons la thèse selon laquelle il faut interpréter de façon globale le phénomène RSE comme une nouvelle configuration, à l'intérieur du mode de production capitaliste, du compromis sociopolitique entre éthique et efficacité. La RSE, n'est ni une nouveauté extraordinaire ni un nouvel avatar du fétichisme capitaliste. Elle est la réponse nouvelle à une nécessité aussi vieille que le capitalisme : celle de donner, pour l'ensemble des acteurs économiques, un sens au processus de production auquel, d'une façon ou d'une autre, ils participent.

Derrière cette question de la responsabilité aujourd'hui adressée à l'entreprise se cache ainsi un problème beaucoup plus général qui a trait à la nature même du mode de production capitaliste. Celui-ci est un mode de production redoutablement efficace en ce qu'il parvient à mobiliser l'essentiel d'une société par un objectif de recherche de puissance productive maximum. Mais il souffre d'un problème intrinsèque bien identifié par Karl Polanyi (1944) : la soumission de la société à la seule logique marchande, dont l'extension caractérise le mode de production capitaliste, pose des problèmes drastiques d'existence même de la société. Une société qui ne serait soumise qu'à des injonctions marchandes visant l'accumulation indéfinie du capital deviendrait en effet l'outil d'une fin qui la dépasserait et du coup se trouverait privée de sens. C'est contre cette absurdité propre au capitalisme que la société se doit, pour exister durablement, d'élaborer des institutions donnant sens au processus de production. Sans réponse à ce problème de la marchandisation, Polanyi pronostique l'effondrement de la société. Il voit, dans le fascisme des années trente, un tel effondrement... et croit percevoir les signes d'un remède à cet effondrement dans la construction, après guerre, de ce qu'on appelé plus tard, le compromis social fordiste.

Reprenant la thèse de Polanyi à notre compte, et suivant pas à pas son analyse du capitalisme, nous cherchons dans cet article à montrer comment, et à quelles conditions, la RSE peut-être porteuse, sur la base et en raison de l'effondrement du compromis social fordiste depuis les années 1970, d'une nouvelle forme de dé-marchandisation socialement indispensable. Les conditions sont institutionnelles, et pour les percevoir clairement, il faut se déprendre de la vision contractualiste standard défendue par Freeman (1984) et souvent étroitement associée, à tort, au phénomène de la RSE. Nous débutons notre article par une présentation des thèses de Polanyi, nous le poursuivons par une mise en perspective historique du phénomène RSE et nous explicitons dans une troisième partie en quoi la RSE doit être institutionnalisée pour avoir un véritable pouvoir régulateur. C'est sur ce point que l'apport de Karl Polanyi, contre la traditionnelle vision contractualiste de Freeman, est décisif. 


\section{Marchandisation fictive et encastrement du capitalisme chez Polanyi}

5 L'apport de Karl Polanyi (1944) à la compréhension du fonctionnement pratique et à la représentation idéologique du capitalisme peut être résumé en quelques idées ${ }^{4}$. Sur le plan pratique, le capitalisme requiert une marchandisation - qui ne peut-être que fictive - de certains piliers de la vie sociale (la terre le travail, la monnaie), ce qui met en péril l'existence même de la société. Sur le plan idéologique ${ }^{5}$, le capitalisme tend artificiellement à réduire l'économique à la stricte maximisation de l'intérêt individuel. Pour Polanyi, l'aboutissement de ce double mouvement, de marchandisation du monde et de réductionnisme individualiste, sape les bases du « vivre ensemble » nécessaire à toute société et, de fait, a provoqué l'anéantissement des sociétés européennes à travers le fascisme. Celles-ci, fortes de cette malheureuse expérience, ont connu leur "grande transformation " à travers la resocialisation du travail, de la terre et de la monnaie, et l'émergence d'une forme de capitalisme ré-encastré socialement. Ce que théorisera plus tard l'École de la Régulation à l'aide du concept de mode d'accumulation fordiste sous régulation monopoliste, rendant ainsi compte de la croissance et de la prospérité des « trente glorieuses » (Boyer, Saillard, 1995).

\subsection{L'irréductible encastrement socio-naturel du capitalisme}

6 L'objet principal de l'ouvrage majeur de Karl Polanyi (1944) est cette grande rupture socio-économique qu'il identifie dans l'histoire récente des sociétés occidentales. Pour lui, dans toutes les sociétés humaines jusqu'au $19^{\text {ième }}$ siècle, le domaine économique est resté confiné dans un statut social finalement subordonné au système des besoins. Quelles que soient leurs formes sociales - lesquelles n'avaient rien de forcément idylliques et pouvaient donner lieu à des relations de domination et à des inégalités -, ces activités se déployaient toujours sous forme sociale encadrée, maitrisée et régulée par des institutions d'ordre juridique ou politique. Les sociétés pré-modernes ont mis en place des dispositifs sociaux complexes pour empêcher que la recherche du profit ne devienne une fin en soi socialement dominante. Jusqu'à l'avènement du marché autorégulateur, il y avait certes des prix et des marchés, mais il s'agissait de prix administrés (ou socialement régulés) et de marchés spécifiques et hétérogènes, ne permettant pas aux pratiques économiques de former une totalité marchande homogène, imperméable aux régulations sociopolitiques et susceptible d'extraversion (Braudel, 1979). Toutefois, dans le sillage de la révolution industrielle, la nécessité de valoriser le capital sans interruption et sur une longue période nécessite que l'on puisse fournir à l'industrie, de manière continue, un flux de travail, de matières premières naturelles, et de monnaie. Le capitalisme industriel nécessite donc la construction d'un pseudo-marché pour ces trois éléments substantiels de la société : la terre (ou plus généralement l'environnement naturel) ${ }^{6}$, le travail et la monnaie. Ce faisant, l'économie marchande se désencastre de la société.

7 Karl Polanyi analyse ce désencastrement à partir de la fiction du marché autorégulateur, que l'idéologie libérale met en scène. Pour lui, le marché désigne le lieu abstrait (théorisé par les économistes classiques et néoclassiques) de formation des prix entendus comme la résultante objective et autonome (par rapport aux forces sociales et politiques) d'une confrontation entre deux flux anonymes (offre et demande, relevant de désir 
hétérogènes). Ce marché autorégulateur est proche de ce que la théorie économique dominante appelle le marché de concurrence pure et parfaite et constitue donc un type de marché particulier en ce que les prix dépendent exclusivement de la concurrence. La relative autonomisation de la sphère économique grâce au développement du marché concurrentiel ne peut bien-sûr être que fictionnel. D'une part parce qu'aucun marché n'existe indépendamment de la société qui le créé : le «marché » n'est pas une réalité naturelle sui generis et ce sont forcément toujours des dispositifs politico-juridiques particuliers qui constituent les conditions de possibilité du marché7. D'autre part, et c'est l'apport conceptuel spécifique de Polanyi, parce que les éléments que le marché autorégulateur suppose de considérer comme des marchandises - le travail, l'environnement naturel et la monnaie - ne le sont substantiellement pas. Pour le comprendre, il est utile de citer longuement Polanyi :

«Le point fondamental est le suivant: le travail, la terre et l'argent sont des éléments essentiels de l'industrie; ils doivent eux aussi être organisés en marchés ; ces marchés forment en fait une partie absolument essentielle du système économique. Mais il est évident que travail, terre et monnaie ne sont pas des marchandises; en ce qui les concerne, le postulat selon lequel tout ce qui est acheté et vendu doit avoir été produit pour la vente est carrément faux. En d'autres termes, si l'on s'en tient à la définition empirique de la marchandise, ce ne sont pas des marchandises. Le travail n'est que l'autre nom de l'activité économique qui accompagne la vie elle-même - laquelle de son côté, n'est pas produite pour la vente mais pour des raisons entièrement différentes -, et cette activité ne peut pas non plus être entièrement détachée du reste de la vie, être entreposée ou mobilisée ; la terre n'est que l'autre nom de la nature, qui n'est pas produite par l'homme; enfin, la monnaie réelle est simplement un signe de pouvoir d'achat qui, en règle générale, n'est pas le moins $\mathrm{du}$ monde produit, mais est une création du mécanisme de la banque ou de la finance d'état. Aucun de ses trois éléments travail, terre, monnaie - n'est produit pour la vente; lorsqu'on les décrit comme des marchandises, c'est entièrement fictif. » (Polanyi, 1983 : 107)

Et il ajoute plus loin cette conclusion :

«(...) aucune société ne pourrait supporter, ne fut-ce que pendant le temps le plus bref, les effets d'un pareil système fondé sur des fictions grossières, si sa substance humaine et naturelle comme son organisation commerciale n'étaient pas protégées contre les ravages de cette fabrique du diable. » (Polanyi, 1983 : 109)

8 Pour Polanyi, le mythe du marché autorégulateur provoque une modification réelle et profonde de nos sociétés au début du dix-neuvième siècle en poussant à la marchandisation, forcément fictive, de l'environnement naturel, du travail et de la monnaie.

\subsection{Le concept de " marchandise fictive »}

Pour saisir en quoi cette marchandisation est fictive, il convient de préciser ce qu'est une marchandise. Polanyi en propose une définition très rapide en emboitant deux critères. « Les marchandises sont ici empiriquement définies comme des objets produits [critère de production] pour la vente [critère de validation]» (Polanyi, 1983, p. 107). Le point essentiel de cette définition est l'existence de ces deux critères qui permettent de distinguer nettement une marchandise de «ce qui circule sur un marché »...car tout ce qui fait l'objet d'un échange marchand n'est pas pour autant une marchandise. On tient là le cœur du concept de marchandise « fictive». Ainsi, ni le travail (au sens de travail-vivant, de force de travail), ni la terre (au sens d'environnement naturel du genre humain) d'une 
part, ni la monnaie de crédit (au sens d'institution sociale rendant possible l'échange marchand) d'autre part, ne peuvent être considérées comme des marchandises. Si on parle de marchandise (ou d'échange marchand) à leur propos, ce ne peut être que par abus de langage, en oubliant de façon plus ou moins explicite l'un ou l'autre des deux critères posés par Polanyi. Elles ne sont cependant pas à mettre tout à fait sur le même plan.

10 A proprement parler et jusqu'à présent, ni l'environnement naturel, ni le travail ne sont les produits d'un processus de production capitaliste. Ce sont des éléments essentiels qui témoignent qu'il y a du donné irréductible dans toute forme humaine de vie sociale. Ce donné naturel et anthropologique, chaque génération le reçoit des précédentes et la transmet aux suivantes. Ce bien commun, qui a été et reste mal mené par la démesure de nos modes de production, est aujourd'hui au cœur des problématiques de développement durable. Le principe d'une humanité produisant toutes les conditions de sa survie amène à adopter sans distance critique l'idéologie prométhéenne qui domine dans les sociétés occidentales ${ }^{8}$.

On peut s'aider de ce qu'ont développé certains théoriciens hétérodoxes contemporains ${ }^{9}$ pour expliciter la définition de la marchandise proposée succinctement par Karl Polanyi. Dans cette optique, le premier critère d'une marchandise, qui pourra sembler évident, est la qualification du contexte économique : on ne peut parler de marchandise que dans le cadre d'une économie de production capitaliste, c'est à dire d'une économie d'initiatives productives privées à la fois décentralisées et interdépendantes. Dans un tel contexte, une valeur d'usage, objet de la transaction sur le marché, peut revendiquer le statut de marchandise au sens strict si elle est d'abord le résultat d'une activité de travail, non pas d'une activité de travail en général, mais d'une activité de travail bien précise, du travail professionnel privé ou marchand (lequel n'est ni le travail domestique, ni le travail des services publics). Cette condition nécessaire n'est cependant pas suffisante: le second critère est que le candidat-marchandise soit validé socialement sur le marché. "Au sens étroit, le concept de marchandise s'applique aux valeurs d'usage présentées sur un marché, au moment effectif de la vente, pour autant qu'elles résultent d'une activité de travail professionnel » (De Vroey, 1984: 111) ${ }^{10}$. Sur cette base, il est possible de donner une explicitation théorique à la notion polanyienne de marchandise fictive. 
Tableau 1 : Le statut particulier des trois marchandises fictives

\begin{tabular}{|c|c|c|}
\hline $\begin{array}{l}\text { Les trois } \\
\text { marchandises } \\
\text { fictives }\end{array}$ & $\begin{array}{l}\text { Premier critère d'existence sociale d'une } \\
\text { marchandise au sens strict : critère de } \\
\text { production pour le marché }\end{array}$ & $\begin{array}{l}\text { Second critère d'existence sociale d'une } \\
\text { marchandise au sens strict: critère de } \\
\text { validation sur le marché }\end{array}$ \\
\hline $\begin{array}{l}\text { Environnement- } \\
\text { support naturel } \\
\text { «La terre } \\
\text { selon Polanyi }\end{array}$ & $\begin{array}{c}\text { Non pertinent } \\
\text { L'environnement naturel a le statut } \\
\text { ontologique d'un donné (et non d'un } \\
\text { produit) que l'on ne peut que modifier } \\
\text { partiellement. Il existe indépendamment } \\
\text { de la transformation permettant d'en faire } \\
\text { le commerce (transformation de } \\
\text { l'environnement naturel en « ressources } \\
\text { naturelles »). } \\
\text { C'estle marqueur fondamental de la } \\
\text { finitude de la condition humaine. }\end{array}$ & $\begin{array}{l}\text { Pertinent } \\
\text { Une fois transformées en ressources par l'effet } \\
\text { du travail humain, des parties de } \\
\text { l'environnement naturel peuvent évidemment } \\
\text { faire l'objet de transactions portant à la fois sur } \\
\text { un volume et sur un prix. C'est done seulement } \\
\text { en vertu du premier critère que l'environnement } \\
\text { naturel n'est pas une marchandise. Il n'est pas } \\
\text { «produit »! }\end{array}$ \\
\hline $\begin{array}{l}\text { Support socio- } \\
\text { anthropologique } \\
\text { « Le travail » } \\
\text { selon Polanyi }\end{array}$ & $\begin{array}{c}\text { Non pertinent } \\
\text { Si le « travail » ne peut pas être considéré } \\
\text { comme un donné naturel, e'est un } \\
\text { construit particulier qui relève } \\
\text { globalement d'un processus de } \\
\text { socialisation extra-économique. }\end{array}$ & $\begin{array}{c}\text { Non pertinent } \\
\text { Sauf à confondre salariat et travail indépendant, } \\
\text { le salarié ne vend aucune marchandise, mais } \\
\text { loue l'usage de sa force de travail dans des } \\
\text { conditions socialement déterminé. Le salaire } \\
\text { n'est pas un prix mais une convention. }\end{array}$ \\
\hline $\begin{array}{l}\text { Support } \\
\text { économique } \\
\text { « La monnaie » } \\
\text { sel on Polanyi }\end{array}$ & $\begin{array}{l}\text { Non pertinent } \\
\text { La monnaie est convention sociétale de } \\
\text { mesure commune des richesses et permet } \\
\text { la pré-validation des productions } \\
\text { marchandes (monnayage et crédit). C'est } \\
\text { une production sociopolitique antéri eure } \\
\text { au champ de la marchandise. }\end{array}$ & $\begin{array}{l}\text { Non pertinent } \\
\text { La théori e keynésienne du taux d'intérêt } \\
\text { comme prix déterminé de manière } \\
\text { conventionnelle-mimétique sur le marché de la } \\
\text { monnaie, et son exposé des conséquences sur le } \\
\text { niveau sous optimal d'investissement qu'un tel } \\
\text { système génère souligne le caractère inadéquat } \\
\text { d'une conception strictement marchande de la } \\
\text { monnaie. }\end{array}$ \\
\hline
\end{tabular}

Sources : adapté de (De Vroey, 1984)

«Fictif» ne signifie donc nullement, pour Polanyi, imparfait. Il se peut au contraire que les marchés "fictifs» fonctionnent exactement comme le conçoit la théorie de la concurrence pure et parfaite. La critique polanyienne n'est pas une critique fonctionnelle dénonçant le fait que, par exemple, le marché du travail ne saurait-être qu'un "quasi » marché en raison du caractère peu flexible du travailleur, ou le fait que le marché ne prendrait pas en compte les externalités négatives de l'exploitation des ressources naturelles ou de la production industrielle. C'est une critique pointant l'artificialisme du traitement marchand de la terre et du travail : ces marchés s'organisent autour de biens et services dont l'existence préexiste à l'échange et qui ne sont donc nullement «produit pour être vendus». Ces marchés son donc «faux» parce que s'y échangent des marchandises «fictives». Or, plus que le simple repérage de cet artifice, ce que Polanyi entend désigner c'est le caractère insoutenable de cette fiction qui si elle n'est pas pris en compte par des institutions spécifiques, provoque la dislocation de la société humaine.

\subsection{La nécessaire institutionnalisation des marchandises fictives... contre le fascisme}

13 Traiter le travail, la monnaie et l'environnement naturel comme s'il s'agissait vraiment de marchandises impacte en effet en profondeur la société et la fait « souffrir ». Non pas bien sûr que le déploiement des forces productives et leur utilisation rationnelle soit mauvaise : bien au contraire produire efficacement est une des conditions essentielles du bien-être social. Mais la contrepartie de cette efficacité, qui consiste à marchandiser des piliers de la vie sociale, est porteuse de menaces. Le marché est en effet une force d'individualisation du social. Il brise les liens collectifs (qui ont besoin de stabilité pour 
s'épanouir) et renvoie les individus à leur propre instrumentalité. Saisis par le marché, les acteurs sociaux abandonnent leur sens de l'éthique et de la raison pratique, pour se comporter comme des automates de marché. La rationalité instrumentale envahit l'espace social.

Contre cette marchandisation les piliers sociaux résistent: ce sont les propriétaires terriens qui veulent obtenir des protections (les fameuses corn laws), ce sont les travailleurs qui se battent pour obtenir des droits les distinguant de la marchandise (c'est l'histoire du mouvement social), ce sont les efforts construits pour bâtir un système monétaire international régulé politiquement. Mais ces efforts s'avèrent vains. La société qui suit la première guerre mondiale est "libérale et marchande ", les forces du marché s'y déchainent sans plus de protection. Plus aucune institution ne permet de faire tenir la fiction de la marchandisation de l'environnement naturel, du travail et de la monnaie. Au point ultime, lorsque la société ne résiste plus à la marchandisation de sa substance, les individus sont littéralement désocialisés, renvoyés à leur simple rationalité économique : "Ainsi, celui qui aurait refusé de reconnaître qu'il agissait seulement par amour du gain serait passé non seulement pour un être immoral mais aussi pour un fou.» (Polanyi, 2002 (1947), : 513). Une société faite d'acteurs renvoyés simplement à la maximisation de leur intérêt est tout simplement invivable : elle dégénère. Cette dégénérescence, Polanyi la voit dans le fascisme: "C'est la réalité d'une société de marché que l'on perçoit dans le totalitarisme» (Polanyi, 2002, : 386).

Cette remarque est d'une sidérante lucidité quant à ce qui unit, au fond, ce que l'observateur pressé a tendance à opposer absolument: la logique libérale et le principe totalitaire (qui ici renvoie au fascisme). En effet, Polanyi perçoit que ce sont là deux figures opposées d'un même malaise social, ou, pour être plus précis, deux stades successifs d'une même maladie consistant à ne plus permettre l'épanouissement d'une forme de raison pratique rendant possible l'établissement de règles de vie communes. À travers le fascisme, les individus désocialisés, incapables d'agir, au sens plein du terme, trouvent un remède à l'anomie en fusionnant dans une commune adhésion à un seul homme et dans l'affirmation de leur lien biologique de race. Que leur reste-t-il d'autre pour faire société ? C'est précisément parce que le totalitarisme est le seul mode d'accord collectif ne supposant pas l'existence d'un consensus social et ne mobilisant pas la raison pratique $^{11}$ qu'il se présente comme l'ultime réflexe d'auto-défense de la société face à la fiction du marché autorégulateur.

La thèse de Polanyi est claire. Elle ne porte pas sur les «failles» ou «dysfonctionnement » du marché. Le système de marché peut fonctionner parfaitement. Mais il suppose une fiction que le marché ne gère pas lui-même et dont surtout il ne gère pas les conséquences. Pour éviter que le capitalisme ne génère des formes de totalitarisme, comme le fascisme, il faut donc aménager et consolider, sur le plan institutionnel, le statut de marchandise fictive. C'est ce que Polanyi croit lire dans «la grande transformation » qui s'opère selon lui au sortir de la seconde guerre mondiale, avec ce que la théorie française de la régulation (Aglietta, $(1987,1988)$; Boyer, (1990, 2002)), appellera plus tard, le modèle d'accumulation fordiste. C'est ce que nous pensons pouvoir lire dans l'émergence de la RSE, que nous analysons comme une des forces compensatoires à l'effondrement de cette régulation fordiste dans les années soixante-dix en Europe. 


\section{La RSE comme réaction à la re-marchandisation néolibérale}

17 Le mouvement de la RSE peut être compris en relation avec la déliquescence du compromis social fordiste (Postel, Sobel, Rousseau, 2006). Le compromis fordiste entre en crise avec l'émergence d'une forme de capitalisme financiarisé (Aglietta et Reberioux, 2004) et en raison de ces limites environnementales qui se font d'abord sentir par la hausse du prix des matières premières auparavant spoliées. La RSE réagit ainsi, d'une part, à la re-marchandisation partielle du travail qui s'opère à la suite de la crise du fordisme dans les années soixante-dix, et, d'autre part, aux premières conséquences visibles de la marchandisation continue de l'environnement naturel, qui se font jour à la même période et qui depuis ne cesse de prendre de l'ampleur.

\subsection{Le compromis social fordiste : dé-marchandisation du travail et de la monnaie...}

18 La grande transformation dont parle Polanyi se produit dans l'immédiat après guerre. Polanyi appelle précisément de ses vœux une modification profonde de l'organisation économique et sociale, qui lui paraît être déjà à l'œuvre, et qui consiste à extraire de la logique du marché le travail, l'environnement naturel et la monnaie.

Concernant la monnaie, l'affaire est d'une certaine manière entendue depuis les accords de Bretton Woods de 1944. Ces accords consacrent en effet l'idée keynésienne d'une monnaie politiquement gérée par le pouvoir politique et mise à l'abri des mouvements marchands par nature "spéculatifs ». Ils initient un système monétaire international fondé sur la convertibilité or du dollar (seule monnaie convertible) et sur l'existence d'un taux de change fixe (mais ajustable par décision souveraine des États pilotant leur Banque centrale) entre le dollar et les monnaies des nations participantes au FMI (France, Allemagne, Grande-Bretagne, Italie...). Un tel système signe la dé-marchandisation de la monnaie dès lors qu'il suppose une gestion politique (concertée) de la valeur relative des monnaies selon le principe des changes fixes, fixe signifiant ici "non flexibles ». Les différentes politiques monétaires nationales déterminent ainsi très largement non seulement le taux de change de leur monnaie, mais plus encore la manière dont les entreprises $\mathrm{y}$ ont accès $^{12}$. Le financement de l'économie est ainsi régulé par des institutions étatiques ou sous contrôle politique. Cette institutionnalisation permet aux États de financer à des taux d'intérêts très faibles (souvent négatifs) les projets d'investissement, qu'ils soient privés ou publics.

Cette dé-marchandisation de la monnaie accompagne une dé-marchandisation du travail, laquelle peut être qualifiée, pour reprendre l'expression de Robert Castel (1995), de « propriété sociale ». En France, dès la Libération, se met en place un processus sociojuridique de consolidation de la condition salariale qui installe le salarié dans une relation de long terme avec son employeur et place cette relation en dehors des relations marchandes traditionnelles. Cette "protection sociale » du travailleur est d'ailleurs au cœur du droit du travail français (Ewald, 1986; Supiot, 1995). Actant que la mise à disposition de la force de travail et la relation de subordination qu'elle suppose place intrinsèquement le travailleur salarié en position de faiblesse, ce droit invente toute une 
série de clauses le protégeant de l'éventuel arbitraire patronal. Au-delà du contrat de travail, les "trente glorieuses ${ }^{13}$ constituent une période durant laquelle les salariés obtiennent, grâce à leurs revendications collectives portées par des syndicats puissants dans les bastions industriels, un ensemble de droits collectifs qui débordent la seule période de travail effectif pour assurer un revenu lors des périodes de chômage, de maladie et de vieillesse (Castel, 1995 ; Friot, 1998) ${ }^{14}$.

21 L'horizon temporel du salarié s'en trouve considérablement consolidé. L'incertitude inhérente aux relations marchandes, lesquelles par définition s'inscrivent dans le temps court et doivent être facilement réversibles, est combattue par la mise en place de dispositifs collectifs qui placent le salariat dans le temps long de la construction de soi. La relation salariale peut ainsi être vectrice d'une forme d'émancipation du salarié, en état de se projeter dans l'avenir, et de concevoir son insertion sociale et plus largement celle de sa famille. À ces dispositions s'ajoutent les services publics, de transport, d'éducation, d'énergie, de poste et télécommunication, ou encore d'eau potable. Ces services intègrent des dispositions favorables aux salariés les plus modestes ne disposant pas de patrimoine financier et favorisent leur insertion sociale au sens plein du terme. Ils permettent en effet aux salariés de se déplacer, de disposer du confort domestique autrefois considéré comme réservé aux nantis, d'assurer à leurs enfants une éducation longue condition d'une promotion sociale ${ }^{15}$. Cette véritable sédentarisation du salariat, dans le temps et dans l'espace social, constitue le ressort principal de la cohésion sociale qui fonctionne relativement bien durant la période des trente glorieuses encore appelée "société salariale » (Aglietta, 1976; Aglietta, Brender, 1984). Conformément à la vision de Polanyi, le capitalisme d'après guerre se "réencastre » à partir d'une véritable mise à distance du marché de la monnaie et du travail. Ce réencastrement est en revanche moins net en matière d'environnement naturel.

\section{2. ...et prédation (non marchande) de l'environnement}

L'environnement reste en effet soumis, quoique de manière souvent indirecte, aux impératifs marchand. Certes l'accès aux ressources naturelles est, dans les pays occidentaux, très largement assuré et régulé par la puissance publique, plus que par le marché. Mais les pouvoirs publics occidentaux s'assurent principalement que cet accès soit le plus large possible, se préoccupant peu des effets qu'une telle prédation des ressources naturelles provoque pour les populations locales du lieu d'extraction (souvent colonisées) ou les générations futures (exclues du débat). L'État se fait ainsi garant de l'accès aux ressources en n'émettant quasiment aucune loi de régulation ou de diminution des pollutions durant toute cette période (Lascoumes, 1993 ; Ost., 1995). Ce principe du large accès recouvre des préoccupations sociales immédiates (le bien-être des populations occidentales) mais aussi et peut-être surtout des préoccupations en terme d'efficacité économique. Les pouvoirs publics se préoccupent en effet essentiellement de la reconstruction et du développement d'une industrie efficace et compétitive: la croissance est en effet conçue (à tort) comme la condition sine qua non de la distribution d'avantages sociaux à même d'alimenter le compromis social évoqué ci-dessus. Dans ce cadre l'environnement est conçu comme un gisement de ressources à même d'alimenter l'industrie d'une part, et comme un élément à mieux maitriser pour assurer certes le bien être social, mais aussi la consommation de biens semi-durables par les ménages. C'est donc une gestion pilotée depuis les intérêts de l'industrie, directement en lui fournissant au plus faible coût possible les ressources dont elle a besoin, ou indirectement, en 
équipant tous les ménages d'un accès aux ressources naturelles (eau, électricité, gaz, essence...) leur permettant de consommer les biens issus des industries de procès au cœur du fordisme. Si l'on reprend la terminologie institutionnaliste de l'École de la Régulation (Boyer, 1986), on peut ainsi parler d'une "forme institutionnelle» spécifique au fordisme consistant en une rente environnementale prélevée sur la nature à des fins de réduction du coût de production des biens et services (Rousseau, 2006 ; Rousseau et Zuindeau, 2007).

Le principe même de ce prélèvement continu, au moindre coût et en fonction des impératifs marchands de la valorisation du capital accumulé, est très directement lié à ce que Polanyi désigne par marchandisation, c'est-à-dire soumission à l'ordre marchand. En ce sens précis, l'environnement naturel est «marchandisé » c'est-à-dire plié aux impératifs marchands de la valorisation du capital. Toutefois la forme particulière (via une organisation publique) que prend la soumission de l'environnement naturel aux nécessités productives régies par l'impératif d'accumulation du capital est très spécifique, et le concept de marchandisation n'est sans doute ici pas parfaitement adapté. Il y a bien "démarchandisation» du rapport à nature. Mais cette démarchandisation renvoie non pas à une promotion et une préservation de l'environnement de l'être humain, mais très étrangement à une accélération de son exploitation et de sa destruction pour les besoins d'un compromis social (qui s'avère donc à courte vue). En ce sens, et pour traduire à la fois le principe d'une gestion non marchande et l'utilisation instrumentale de la nature comme simple «support » de la valorisation marchande du capital) nous proposerons de qualifier le rapport à la nature entretenu dans le fordisme de "prédation». Une prédation construite publiquement pour alimenter le compromis social et permettre simultanément l'accumulation du capital. Une prédation qui suspend cependant l'évaluation marchande (illusoire) de ce que valent les ressources naturelles privant par la même les pays détenteurs de ces ressources (pays souvent colonisés) de toute possibilité d'en monnayer leur utilisation.

\subsection{Crise du fordisme et processus de re-marchandisation}

24 Ce mode d'accumulation entre en crise dans les années soixante-dix. Cette crise présente plusieurs dimensions : une crise financière, sociale, commerciale et environnementale (Boyer, 2004 ; Postel, Rousseau, Sobel, 2006). Il n'entre pas dans l'objet de cet article d'analyser avec précision les relations complexe entre ces différents aspects de la crise du fordisme. Mais, pour l'essentiel, on peut les relier en soulignant le processus de fond qui est à l'œuvre, celui d'une triple re-marchandisation: la re-marchandisation de la monnaie, re-marchandisation du travail, et construction d'un dispositif marchand d'usage des ressources environnementales.

La re-marchandisation de la monnaie se joue dans la crise du SMI dès 1971. La période 1971-1973 marque la fin du système monétaire mis en place à Bretton Woods, sur l'initiative du président Nixon qui suspend la convertibilité or/dollar. Elle se traduit en 1973 par l'avènement du flottement généralisé des monnaies. Les accords de la Jamaïque en 1976 officialisent cet abandon du système des changes fixes. Ce système suppose une évaluation permanente des monnaies en fonction des performances du système économique qu'elles couvrent, et en particulier en fonction de la rentabilité du capital accumulé par ces économies: autrement dit, dans la terminologie polanyienne, une marchandisation. 

années quatre-vingt à travers la remise en cause progressive du contrat de travail à durée indéterminée comme norme salariale, puis par le durcissement, en Europe des conditions d'accès aux différentes protections et allocations sociales (Husson, 2003 ; Castel, 2003). Les récents débats autour de la nécessaire modernisation/flexibilisation du marché du travail, avec ou sans contrepartie sécurisant les parcours professionnels (Ramaux, 2006), montrent d'une part qu'il s'agit bien de replacer la relation salariale sous l'égide du seul marché, d'autre part que ce processus bien qu'ancien n'a pas encore parfaitement abouti en Europe, et peut-être plus particulièrement en France. Ces deux mouvements, de financiarisation d'une part et de flexibilisation de l'autre, sont liés: les entreprises arguent en effet que face à un actionnariat mouvant et en état de mettre en concurrence les différents lieux de production dans le monde, confrontées de plus à une concurrenceprix réactivée sur le marché des biens et services, elles ne peuvent plus garantir une relation de long terme aux salariés, sans mettre en péril leur activité.

La construction progressive d'un marché des ressources environnementales est lent et complexe, en ce qu'il se traduit par deux phénomènes distincts: montée de l'encadrement réglementaire de l'usage de l'environnement (comme ressource et comme réceptacle des pollutions industrielles) et construction (sous l'égide de l'OMC) d'une organisation internationale marchande de l'exploitation de l'environnement. Les principes prédateurs qui sous-tendaient la rente environnementale sont remis en cause et l'enjeu devient alors pour les entreprises de parvenir à acquérir des positions stratégiques leur permettant d'assurer leur activité productive au-delà des aléas marchands et de la hausse possible du prix de certaines ressources sous l'effet de leur raréfaction réelle et/ou organisé par la juridiction environnementale naissante.

\subsection{L'émergence de la RSE sous une double nécessité de résister à la marchandisation}

On peut interpréter l'émergence de la RSE comme une réaction au processus de remarchandisation qui suit l'entrée en crise du compromis fordiste - pour parler comme l'École de la régulation - ou de la grande transformation - pour parler comme Polanyi. Cette émergence s'appuie sur deux mouvements distincts dans leur intention et qui se rejoignent de manière relativement fortuite. Le premier mouvement est propre à l'entreprise et s'explique par des considérations d'ordre stratégique à moyen terme : l'entreprise a en effet besoin de stabiliser son environnement immédiat pour limiter les risques financiers, productifs et commerciaux. Mais ce premier mouvement, stratégique, en rejoint un second que l'on peut qualifier de sociopolitique. Ce dernier s'inscrit dans le cadre de la nécessaire légitimation, par le capitalisme, de son mode de fonctionnement. Chiapello et Boltanski (1999) ont analysé avec finesse la nécessité, déjà repérée par Weber, pour le capitalisme de justifier le principe de l'accumulation du capital par l'existence de retombées jugées positives par la collectivité. Les processus de RSE sont portés par cet objectif de légitimation.

L'engagement des entreprises dans des processus de RSE peut recevoir une explication purement stratégique. Cet engagement s'explique en effet par la nécessité dans laquelle elles sont de retrouver de la stabilité au sein des relations fondamentales que noue l'entreprise avec le marché des capitaux, du travail, des ressources naturelles et des biens. D'une certaine manière, l'entreprise cherche, toujours, à domestiquer le marché, 
c'est-à-dire à substituer des relations de confiance durables à des relations marchandes immédiatement réversibles ${ }^{16}$. Le marché ne permet en effet pas à l'entreprise de garantir avec suffisamment de certitude à long terme le débouché des produits, la pérennité de l'investissement et la mise à disposition des matières premières et d'une force de travail compétente. L'analyse économique a bien explicité les enjeux de ces exigences. Keynes a depuis longtemps souligné la difficulté pour l'entreprise de devoir faire face au caractère volatile du financement par les marchés financiers. On sait aussi depuis les travaux menés sur la segmentation du marché du travail (Michon, Petit, 2007) que la mise à disposition d'une main-d'œuvre immédiatement compétente et capable de s'insérer dans l'entreprise n'est pas un service marchand. C'est également l'argument de Williamson (1983) et du courant de recherche néo-institutionnaliste : le mode de production capitaliste met en jeu des investissements irréversibles, en particulier en matière de main-d'œuvre, ce qui nécessite de faire sortir la relation d'emploi du simple marché pour le faire basculer sous le registre de la relation d'autorité durable permettant l'existence d'un apprentissage de nature idiosyncrasique ${ }^{17}$. Il est donc nécessaire pour l'entreprise de disposer d'un noyau de salariés stable et accédant au langage commun propre à l'entreprise. Cette nécessité ne concerne cependant pas avec la même intensité toutes les couches du salariat, une large part des salariés, qui effectuent des tâches relativement standardisées étant souvent considérés comme interchangeables. Pour ce qui concerne les débouchés, là encore les pratiques de fidélisation de la clientèle sont un grand classique du fonctionnement concret du capitalisme. De ce point de vue, les démarches de RSE représentent simplement un approfondissement du principe de la marque. L'entreprise cherche depuis toujours à faire basculer la relation de clientèle vers une forme de relation marquée par un attachement dépassant le simple échange marchand.

Le rapport à la nature quant à lui constitue en revanche un rapport bien peu pris en compte par l'entreprise depuis les origines du capitalisme industriel. La prise de conscience des limites prévisibles de l'approvisionnement en ressources naturelles date en effet des années soixante-dix. Elle est consécutive à la hausse simultanée du prix des matières premières et à celle de la constatation puis la contestation sociale des pollutions industrielles (Clerc, Lipietz, Satre-Buisson, 1985). Les entreprises sont ainsi conduites à anticiper doublement sur ses conséquences. D'une part, l'entreprise anticipe sur la raréfaction et donc le surenchérissement de son accès aux ressources naturelles, ce qui lui permet de mettre au point des scénarii alternatifs et de s'adapter plus rapidement lorsque les premiers signes de tension se manifestent. D'autre part, l'entreprise se doit aussi d'anticiper le durcissement de la réglementation en ce qui concerne non plus l'approvisionnement mais les retombées écologiquement néfastes de son activité. Les pouvoirs publics des nations capitalistes avancées se sont progressivement saisis de ces questions sous l'angle de la santé publique et de la pérennité de l'activité ${ }^{18}$. L'entreprise a donc tout intérêt d'une part à anticiper ce durcissement, voire à l'orienter en suggérant, par son comportement, la mise en place de dispositifs peu coercitifs, ou n'entrant pas directement en contradiction avec ces objectifs productifs. Sous l'angle stratégique, la veille environnementale et la recherche de stratégies productives durables sont donc parfaitement cohérentes avec l'objectif de maximisation du profit à moyen terme.

31 Au total, sur le plan stratégique, il s'agit donc pour l'entreprise de combattre toute une série de risques: le risque commercial en fidélisant ses clients; le risque financier en fidélisant ses actionnaires; le risque social en prévenant les conflits productifs et en assurant une culture commune en son sein ; le risque environnemental en adaptant son 
comportement et son mode de production avant que la raréfaction des ressources productives soit effective; le risque juridique en cherchant à anticiper et à orienter l'action du législateur en amont par la mise en place de comportements ayant valeur d'exemple ou d'accords collectifs d'entreprise anticipant sur la loi future ${ }^{19}$. La remise en cause des institutions régulatrices du fordisme, et la re-marchandisation du travail et de la monnaie, a pour conséquence la nécessité de mettre en place au plan microéconomique des dispositifs la protégeant du marché. C'est là un premier moteur, fondé sur la rationalité stratégique, de l'émergence du management participatif, puis des processus de RSE.

Cette impulsion, qui part des comportements et intérêts privés des entreprises, est relayée par un second moteur, sociopolitique, qui tient à la nécessité qu'a le capitalisme de s'auto-justifier pour éviter son délitement. Cela explique l'émergence d'une forme de prosélytisme dans le champ de l'éthique d'entreprise. Celle-ci n'est en effet pas seulement portée par des entreprises individuelles cherchant à posséder un coup d'avance relativement à leurs concurrentes, mais est l'objet d'un soutien des institutions nationales (par exemple en France la loi NRE), internationales (rôle de commission européenne, du BIT ou de l'ONU) et plus encore des multiples réseaux d'entreprises et d'associations qui défendent les pratiques RSE et cherchent à normer ces pratiques ${ }^{20}$. Ce second moteur trouve place dans ce que Boltanski et Chiappello (1999) qualifient de " nouvel esprit du capitalisme ». Les différents acteurs s'entendent en effet pour, d'une part, reconnaître la légitimité d'une forme de capitalisme déliée de la contrainte nationale et $\mathrm{du}$ principe de l'engagement irréversible, en opposition aux principes du fordisme, et pour, d'autre part, parvenir à insérer suffisamment les individus dans des réseaux sociaux leur permettant de valoriser leur expérience et capitaliser leurs apprentissages. Cette nouvelle forme de capitalisme prétend ainsi concilier une liberté nouvelle (vis-à-vis de l'État en particulier) et une légitime sécurité à travers ce réseau des pairs dans lesquels les individus devraient s'insérer. Elle peut séduire les individus, membres d'une élite productive de cadres mondialisés, soucieux de liberté et agacés par les différents freins réglementaires mis à leur liberté économique (de production ou de travail). Elle trouve aussi un écho certain auprès des États débordés par la puissance de la finance mondialisée et relativement heureux de quitter le champ de la régulation autoritaire pour laisse faire les acteurs. Elle trouve enfin un appui auprès des entreprises, soucieuses de se délier des engagements de long terme contractés à l'époque fordiste. Dans cette optique, le mouvement de la RSE arrive à point nommé puisqu'il cherche précisément à resocialiser les liens marchands, tout en conservant le principe d'un capitalisme mondialisé dominé par une finance dérégulée. D'une certaine manière, les intérêts stratégiques des différentes entreprises et les nécessités politiques de trouver une assise sociale au capitalisme se trouvent donc mis en cohérence.

\section{La RSE : une voie étroite qui nécessite une institutionnalisation}

33 À l'aune d'une perspective institutionnaliste polanyienne, le mouvement de la RSE apparait donc comme une réaction à la re-marchandisation du «travail » d'une part, et aux effets désormais visibles de l'usage marchand de «l'environnement naturel » d'autre part $^{21}$. Toutefois, la manière dont sont saisis les enjeux de cette double remarchandisation fictive est pour le moins complexe. La RSE ne remet en effet 
formellement en cause ni le principe d'une marchandisation accrue de la monnaie (Aglietta, Rébérioux, 2006), qui signe le retrait de l'État régulateur et des partenaires sociaux, ni celui d'une gestion, par le marché, du travail et des matières premières. Elle compte simplement sur les différents acteurs concernés - ce qu'il est désormais convenu d'appelé « les parties prenantes »- pour élaborer des conventions de mise sur le marché qui garantissent qu'au-delà de leur évaluation marchande, les éléments de la nature et le travail humain mis sur le marché le sont dans des conditions «légitimes " ${ }^{22}$. En ce sens, elle choisit une voie étroite dont l'ouverture suppose l'activation de soutiens institutionnels à ce processus en opposition à la logique contractualiste qu'il l'a popularisée.

\subsection{Une démarchandisation partielle menée sous le régime d'une monnaie marchandise?}

La clé de voûte du modèle fordiste réside dans la mise en place, à Bretton Woods d'une gestion institutionnalisée (et donc non marchande) de la monnaie. C'est aussi d'abord par ce biais que cette première forme de démarchandisation entre en crise. Or, étrangement, le phénomène de la RSE n'entend nullement revenir sur ce cadre macroéconomique général qui voit la marchandisation de la monnaie permettre aux détenteurs de capitaux de disposer d'un formidable levier sur les États et les entreprises. Au contraire, on peut penser que la RSE apparait aux yeux de ces promoteurs comme la solution sociale et environnementale à adopter dans un capitalisme financiarisé se caractérisant par la réduction des marges de manœuvre des États. De fait la question de la régulation monétaire et financière nécessite, comme l'actualité récente nous le démontre, la mise en place de solutions à la fois coordonnées internationalement et situées à l'échelon macroéconomique. Elle se situe bien au-delà des capacités des acteurs individuels.

Là se situe, précisément, le premier pari sur lequel reposent les mouvements de RSE : parvenir à réintroduire des préoccupations éthiques sous l'égide d'une finance dont la puissance n'est nullement remise en cause (jusqu'à une période récente et encore bien incertaine). La logique de ce pari consiste à faire en sorte que les acteurs auxquels la marchandisation de la monnaie donne le principal pouvoir économique, c'est-à-dire les détenteurs de capitaux, modifient leur comportement et agissent en intégrant dans leurs évaluations des préoccupations " éthiques " (portant sur la préservation des ressources naturelles et des équilibres sociaux). Les premiers travaux sur l'une des mises en application de ce pari, dans le champ de l'investissement socialement responsable, font montre d'un certain pessimisme (Dejean, 2005). Il semblerait donc que l'espoir de voir, par les mouvements de RSE, triompher une forme de "démocratie actionnariale » soit illusoire (illusion déjà dénoncée par Lordon (2000)). Mais cette pression directement exercée sur les actionnaires n'est en fait qu'un des deux niveaux d'action que comprennent les phénomènes de RSE. Le second prend acte de la domination de la sphère financière, et fait le pari d'une sensibilité de cette sphère à celle des biens et services, et, plus précisément à l'attention que portent les détenteurs de capitaux aux désirs et attachements des consommateurs. C'est dans cette instrumentalisation de l'échange à des fins éthiques que réside le pari de la RSE. 


\subsection{Une démarchandisation menée...dans le jeu même de l'échange}

36 Le second niveau auquel opèrent les processus de RSE est donc celui de l'échange marchand lui-même. C'est en effet paradoxalement à travers l'échange même que la RSE porte l'espoir d'une démarchandisation des piliers de la vie sociale que sont l'environnement naturel et le travail humain. La RSE ne prétend jamais, en effet, sortir entièrement du marché la relation au travail et à la nature. Elle suppose que l'on ne réduise pas la relation salariale à une relation purement marchande... mais ne remet pour autant en cause ni le principe d'une évaluation marchande de la valeur du travail, ni le principe de la flexibilité qui caractérise la re-marchandisation du travail. De même en matière environnementale, les processus de RSE ne dénoncent jamais le principe de la propriété privée ou la libre-circulation des marchandises agricoles.

C'est que la RSE joue sur le contenu des conventions gérant la mise sur le marché23. Il en va ainsi notamment des engagements dans le champ du commerce équitable, ou du respect des principes de l'OIT ${ }^{24}$, qui donnent un cadre global à la relation d'emploi, sans pour autant entrer dans le détail de sa rémunération ou de son encadrement, comme c'est le cas dans les conventions collectives de branches des régulations nationales fordistes. Il en va de même en matière environnementale. Ce n'est pas le principe même d'une évaluation marchande des matières premières qui est mis en cause, mais la manière dont la mise sur le marché est opérée, avec ou sans prise en compte de la durabilité de la ressource ou des retombées négatives de sa consommation. Cette attention, réelle ou supposée, au devenir de la ressource naturelle conduit les entreprises responsables à consommer des marchandises issues de filières de production raisonnées ou biologiques, assurant non seulement la mise à disposition du bien, mais encore le fait que cette mise à disposition n'entrave pas la production future de biens identiques (par épuisement de la biosphère) ou n'altère pas la qualité environnementale de l'espace où elle est produite (par destruction d'une partie de la biosphère). L'essor des produits normés, souvent à l'initiative des entreprises elles-mêmes, montre l'importance que prend cette dimension «sociale » de la marchandisation fictive de l'environnement.

Sur cette question, le concept à mobiliser est donc celui de convention de qualité (Eymard-Duvernay, 1989, 2006). De la même manière que l'on a pu souligner ${ }^{25}$ que la rémunération du travail formait une convention bien plus qu'un prix - ce que remet en partie en cause le capitalisme financiarisé - on peut souligner que le prix des matières premières forme également une convention dans la mesure où il dépend de la manière dont ces matières sont extraites de la nature qui les met à disposition gratuitement. L'économie des conventions a largement investi le champ de la mise à disposition des biens et services en démontrant la faiblesse théorique du concept de marchandise, lorsqu'il ne renvoie qu'à des processus d'achat et de vente (Favereau, Biencourt, EymardDuvernay, (2002) $)^{26}$. Plus généralement, le champ de la socio-économie met en avant le principe de la construction des marchés et de l'accord social qui préside à la mise à disposition des biens. Un accord social est souvent tacite, quelque fois réglé par la loi, et plus ou moins développé.

39 Le mouvement de la RSE s'engouffre dans cet espace jusqu'alors peu mobilisé politiquement qui sépare l'existence d'une force de travail ou d'une ressource naturelle (toutes deux étant, rappelons-le, indépendantes du marché) et leur mise sur le marché permettant de leur attribuer un prix. Cette démarche peut-être qualifiée de 
conventionnelle dans la mesure où elle s'appuie sur une forme d'accord collectif qui peut être tacite, qui facilite la coordination, sert ainsi l'intérêt de chacune des parties, et, ce faisant, ne requiert pas l'accord explicite de tous les participants (Postel, Rousseau, 2008). Nécessaires, contingentes, autorenforçantes, ces conventions sont aussi signifiantes : elles traduisent une visée éthique commune à l'ensemble des acteurs, dans la mesure bien-sûr de l'information dont ils disposent. La question du caractère solide et effectif de l'encastrement social des deux marchandises fictives que permet la RSE dépend alors de la nature du processus de production de ces conventions de mise sur le marché et des acteurs qu'il mobilise. Autrement dit, la RSE vise d'une certaine manière à jouer l'échange marchand contre le marché lui-même, en accrochant l'échange entre acteurs à des institutions extérieures contenant des valeurs et références communes portant une certaine vision du monde ${ }^{27}$. C'est de la qualité de ces ressorts institutionnels qui se manifestent dans l'échange que dépend donc le potentiel régulatoire de la RSE. Une "qualité » institutionnelle qui porte sur la nature éthique des règles de définition des marchandises plutôt que sur la simple organisation des échanges.

\subsection{Pour une critique de l'approche contractualiste standard}

L'affirmation de cette dimension institutionnelle de la RSE ne va pas de soi. Au contraire, il est fréquemment acté, sans discussion, que la RSE désigne précisément la possibilité d'une régulation spontanée, autonome, ne requérant aucune institution. C'est la thèse que développe avec un très grand succès Freeman $(1984,1994)$ à travers la théorie dite des «parties prenantes» (ou «stakeholders ») $)^{28}$. Dans un article collectif, Freeman salue ainsi l'émergence d'un nouveau capitalisme, le "capitalisme des parties prenantes ». Celui ci se caractérise par la disparition d'une médiation collective entre acteurs, et en particulier par la disparition de toute forme étatique. Celle-ci, successivement justifiée pour des raisons sociales (par Marx), économiques (par Keynes), entrepreneuriales (par Schumpeter), morales (par Friedman, qui souhaite protéger les actionnaires). Désormais, cela serait devenu inutile, les individus ayant pris conscience de leur capacités éthiques pourraient s'auto-organiser :

\footnotetext{
«De la valeur peut être créée, vendue et maintenue dans le temps, parce que (1) des stakeholders peuvent joindre de manière satisfaisante leurs besoins et leurs désirs en passant des accords volontaires entre eux qui sont pour la plupart tenus (2) parce que toutes les transactions impliquent des clients, des fournisseurs, des employés et de financiers, (3) parce que les stakeholders acceptent leur responsabilité, (4) parce que les humains ont des motivations et des valeurs différentes, (5) parce que les stakeholders sont motivés par leur valeurs ».(Freeman, Martin, Pamar : 311).
}

41 Pour Freeman, il ne s'agit pas de constater un mouvement historique contingent, mais de hâter ce qui lui apparait comme une modification profonde du «capitalisme » vers une forme meilleure. Un capitalisme qui ne serait plus fondé sur la concurrence et l'atomicité mais sur la recherche permanente d'arrangement entre contractants, sur le modèle des relations entre entreprises. Le capitalisme qui se dessine est alors un capitalisme de la responsabilité individuelle et de la négociation. C'est, pour Freeman, un capitalisme postconflit social ${ }^{29}$, et donc post-État (dans la veine des réflexions sur la fin de l'histoire) qui s'oppose à la représentation marxo-keynésienne. Mais pour Freeman, cette forme de capitalisme se distingue aussi de la représentation néoclassique fondé sur le mythe d'un marché autorégulateur rassemblant des individus atomisés et exclusivement tournés vers le principe de la maximisation des intérêts (ou plus généralement l'accomplissement des 
visées qui peuvent être désintéressées). On sait en effet qu'un marché de ce type, un marché walrassien, requiert toujours, a minima, une institution centralisatrice : celle du commissaire priseur (Berthoud, 1988). Contre cette vision le capitalisme des parties prenantes représente l'émergence d'un capitalisme a-institutionnel, ne reposant que sur la liberté et l'éthique d'individus, libre, égaux et portés vers la discussion ${ }^{30}$.

Une telle représentation $d u$ capitalisme est cependant parfaitement factice. Le capitalisme ne saurait être a-institutionnel, précisément parce qu'il repose d'abord sur la marchandisation fictive des piliers de la vie sociale en vue de l'accumulation du capital. Dans un capitalisme sans règle, cette logique s'impose donc sans retenue. D'une certaine manière, donc, le raisonnement de Freeman nie la logique même du capitalisme. Si l'on veut bien suivre Polanyi, cette logique n'est pas celle d'un conflit d'appropriation entre acteurs, que des contrats interindividuels équitables (dans un régime de concurrence pure et parfaite organisé par des règles de droit) pourraient résoudre. C'est un problème structurel très en amont de la négociation marchande qui pose le problème de l'articulation de la logique sociale éthico-politique et de la logique productive instrumentale. Un problème de désencastrement de l'économique, imposant sa logique destructrice à la société par la marchandisation de ces piliers, que des contrats interindividuels ne peuvent résoudre. Réarmer les dimensions sociales et environnementales contre la seule logique économique de l'efficacité et de l'accumulation du capital requiert précisément des institutions collectives en dehors des contrats et du régime de l'organisation marchande. Des institutions gérant l'articulation de l'économique et du social à partir de la prise en compte de la nature particulière des «marchandises» fictives. C'est ce à quoi peuvent contribuer les démarches de RSE en générant des conventions communes préfigurant de futures règles collectives permettant de mettre à distance du marché la « terre » et le « travail » (pour ce qui est du champ de la RSE). Les démarches et débats autour de l'entreprise responsable, et plus précisément autour des normes qui encadrent ces dispositifs ouvrent un espace délibératif et contribuent ainsi à préserver la possibilité de la «raison pratique » contre celle de la stricte rationalité instrumentale ${ }^{31}$.

\section{Conclusion}

$\mathrm{Au}$ contraire du rêve contractualiste que l'on trouve dans les approches en termes de parties prenantes, la RSE ne peut exister qu'en contribuant à la construction d'institutions communes organisant de manière collectivement légitimée le rapport au travail et à la nature. Si les processus de RSE n'étaient qu'une manière de repousser, comme le suppose la théorie de Freeman, tout dimension de régulation collective, alors le risque serait très élevé d'y voir un véritable péril visant à supprimer toute régulation collective. Faire appel à la RSE contre l'existence de régulations collectives, c'est en effet non pas seulement prendre le risque que la RSE ne soit qu'un faible mouvement sans contenu... mais surtout réunir les conditions pour que la RSE serve de prétexte à l'élimination des derniers verrous qui tiennent encore le travail et l'environnement naturel hors d'une régulation purement marchande. Comme le souligne supiot (2007 : 34) : «Il faut donc se défaire des illusions du "tout contractuel". Loin de désigner la victoire du contrat sur la loi, la "contractualisation de la sociéte" est bien plutôt le symptôme de l'hybridation de la loi et du contrat et de la réactivation des manières féodales de tisser le lien social 
». Derrière l'idéologie contractualiste, portée par Freeman, se profile donc le retour au féodal, pour Supiot, ou, à la dislocation sociale, pour Polanyi.

Au contraire la RSE, qui vise à une gestion «durable» du travail et des ressources environnementales, peut être porteuse d'une véritable forme ré-encastrement de l'économique sous une domination qui reste, rappelons-le, capitaliste. En terme polanyien, la RSE vise à ne pas considérer le travail et les ressources naturelles comme de simple «marchandises » et consiste à réinterroger le statut de ce que cet auteur qualifie de «marchandises fictives ». Certes, en choisissant de le faire depuis l'échange lui-même et à partir de la sensibilisation du client, la RSE choisit une porte étroite. Elle n'est cependant pas forcément vaine, si l'on s'accorde à l'orienter vers l'existence d'accords communs contraignants au lieu de la circonscrire à un simple engagement volontaire individuel. En choisissant de le faire à partir d'un engagement volontaire des acteurs majeurs de capitalisme, selon un mode de régulation fondé sur une convention (librement consentie, donc, mais dotée d'une forme normative indéniable une fois légitimée), les processus de RSE font le pari que les rapports de force entre ces acteurs - clients, actionnaires, salariés, ONG environnementales - permettront de produire des institutions équilibrées. C'est un pari incertain, mais qui sans doute, dans un univers qui se caractérise par la faiblesse des médiations politiques, mérite attention.

Penser la RSE comme un processus devant s'institutionnaliser, chercher à fournir aux acteurs de ces processus des repères collectifs ayant un contenu contraignant : ce sont les deux conditions sine qua non pour que la RSE soit, véritablement, porteuse d'une forme de ré-encastrement social des deux marchandises fictives que sont les ressources naturelles et le travail. C'est à ses deux conditions qu'elle pourra, avec d'autres dispositifs sociopolitiques, être une forme de défense et de promotion du «développement durable».

\section{BIBLIOGRAPHIE}

Aglietta M., (1997, 1976), Crise et régulation du capitalisme, Odile Jacob, Paris

Aglietta M., Orléan A., (1982), La violence de la monnaie, Presses Universitaires de France, 1998

Aglietta M., Brender A., (1984), Les métamorphoses de la société salariale, Calmann-Lévy, Paris

Aglietta M., Reberioux A., (2004), Dérives du capitalisme financier, Paris, Albin Michel

Aubenque P., (1963), La prudence chez Aristote, PUF, coll. « Quadrige », Paris, 2004 (4édition)

Arrow K. J., 1973b, “Information and Economic Behavior", lecture to the Federation of Swedish Industries. Publié dans The Collected Papers, vol 4, p. 136-152

Boidin Bruno, Postel Nicolas, Rousseau Sandrine, (2009), La responsabilité sociale des entreprises, Une perspective institutionnaliste, Lille : Editions du Septentrion

Berthoud A. (1988) «Économie politique et morale chez Walras », đEconomia, série PE de la revue Economie et Société, $n^{\circ}$ 9, p. 65-93 
Boltanski L. et Chiapello E., (1999), Le nouvel esprit du capitalisme. Paris, Gallimard Boyer R., (1986), Théorie de la régulation. Une analyse critique, La Découverte, Paris Boyer R., (1989), «Justice sociale et performances économiques : de la synergie au conflit », in Affichard J., De Foucauld J.B. (eds), Justice sociale et inégalités, Seuil, Paris

Boyer R., (1995), «Vingt ans de recherches sur le rapport salarial : un bilan succinct ", La théorie de la régulation L'état des savoirs (sous la direction de Boyer R. et Saillard Y.), La découverte, Paris Boyer R., Saillard, Y. (1995), La théorie de la régulation L'état des savoirs, La découverte, Paris Boyer R., (2004), « Une théorie des crises », chapitre IV de Théorie de la régulation 1. Les fondamentaux, Repères, La Découverte, Paris, p. 75-103

Braudel F., (1979), Civilisation matérielle, économie et capitalisme XV-XVIII siècle - Tome 2 les jeux de l'échange, Armand Colin, Paris

Cartelier J., (1983), « Une introduction à l'économie hétérodoxe », Working Paper, n 8302, Université Catholique de Louvain

Castel R., (1995), Les métamorphoses de la question sociale, Fayard, Paris

Castel R., (2003), L'insécurité sociale, Le Seuil, Paris

Capron M., Quairel-Lanoizelée F., (2004), Mythes et réalités de l'entreprise responsable, Paris, La Découverte

Capron M., (2007), La responsabilité Sociale de l'Entreprise, Coll. Repère, Paris la découverte

Cartelier, J., (1996), La monnaie, Dominos, Flammarion, Paris

Cazal D., (2008), « Parties prenantes et RSE : des enjeux sociopolitiques au-delà des contrats », Revue de l'Organisation Responsable, $\mathrm{n}^{\circ} 6$

Clerc D., Lipietz A., Satre-Buisson J., (1985), La crise, Syros Alternatives, Paris

Coase R, 1960, Coase R., (1960), "The problem of social cost", Journal of Law and Economics, 3, oct., p. $1-44$

Defalvard H. (1994), Essais sur le marché, Paris, Syros

Déjean, F., (2005), L'Investissement Socialement Responsable - Étude du cas français, Paris, Vuibert

Defalvard H., (1994), Essais sur le marché, Paris, Syros

Deleplace G., (1979), Théorie du capitalisme : une introduction, Grenoble, PUG Maspero

De Vroey M., (1984), « Marchandise, société marchande, société capitaliste : un réexamen de quelques définitions fondamentales », Cahiers d'économie politique, n 9, p. 109-134

Eymard-Duvernay F. (Sous la direction de), (2006), L'économie des conventions Méthodes et résultats Tome 1 Débats, La Découverte, Paris

Esping-Andersen G., (1999), Les trois mondes de l'Etat-providence, PUF, Paris

Eymard-Duvernay F., (1989), « Convention de Qualité et formes de Coordination », L'Economie des Conventions, Revue Economique, p 329-359

Ewald F., (1986), L'État-providence, Paris, Grasset

Favereau O., Biencourt O., Eymard-Duvernay F., (2002), "Where do market come from ? From (quality) conventions !", in Conventions and Structures in Economic Organization : Markets, Networks, and Hierarchies, Favereau O., Lazega E. (eds), Cheltenham, Edward Elgar Publishing 
Freeman R.E., (1984), Strategic Management : A Stakeholder Approach, Pitman, Boston

Freeman R.E., (1994), "The politics of stakeholder theory : some future directions", Business Ethics Quaterly, 4 (4), p. 409-421

Freeman R.E., Kirsten M., Bidhan P., (2007), “Stakeholder capitalism”, Journal of Business Ethics, 74, 303-314

Friot B., (1998), Puissances du salariat, La Dispute, Paris

Guttman R., (1995), « Monnaie et crédit dans la théorie de la régulation », in Théorie de la régulation L'état des savoirs, Sous la direction de Boyer R. et Saillard Y., La Découverte, Paris, p. 85-104

Habermas J., (1983), Théorie de l'agir communicationnel, (Trad. de J. M. Ferry), Paris, Fayard Husson M., (2003), Les casseurs de l'Etat social Des retraites à la Sécu : la grande démolition, Paris, La Découverte

Larrère C., (2000), Philosophie de l'environnement, PUF, Paris

Lascoumes P., (1993), «La "nature" comme intérêt protégé par le droit pénal. Les trois étapes d'un parcours incertain ", in Image set usages de la nature en droit, Gérard P., Ost F., van de Kerchove M. (Eds.), Publications des Facultés Universitaires Saint Louis, Bruxelles, p. 209-230 Lavoie M., (2004), L'économie post-keynésienne, La Découverte, Paris

Lengaigne B., (2009), « Les usages contemporains de la notion de "partie prenante" : entre contrat, risque et responsabilité » in Boidin et ali (dir), pp. 55-67

Lordon F., (2000), Fonds de pension : piège à con?, Liber Raison D’agir

Maucourant J., Servet J.-M. et Tiran, A., (1998), « La réception de la pensée de Karl Polanyi », in Maucourant J., Servet J.-M, Tiran A. (dir.) La modernité de Karl Polanyi, Paris, L’Harmattan, p. $385-398$

Maucourant J., (2005), Avez-vous lu Polanyi ?, Paris, La dispute

Michon F. et Petit, S. (Coord.), (2007), Is the concept of labour market segmenation still accurate ?, $\mathrm{n}$ - spécial, Economies et Sociétés - Série Socio-Economie du travail, 28, juin

Mercier S. (2006), « La théorie des parties prenantes : une synthèse de la littérature », in Bonnafous-Boucher M., Pesqueux Y., p. 157-172

Moulier-Boutang Y., (1999), De l'esclavage au salariat, économie historique du salariat bridé, Paris, PUF Ost F., (1995), La nature hors la loi, l'écologie à l'épreuve du droit, éd. La Découverte, Textes à l'appui, Série Ecologie et Société

Peters B. G., Pierre, J. ; (1998), “Governance without government ? Rethinking public administration", Journal of Public Administration Research and Theory, 8(2), 223-243

Plihon D., (2004), Le nouveau capitalisme, Repères, La découverte, Paris

Polanyi K., (1983), La Grande Transformation, Aux origines politiques et économiques de notre temps ( $1^{\text {re }}$ édition anglaise, The Great Transformation, 1944)

Polanyi K., (1957), « L'économie en tant que procès institutionalisé », in Polanyi K., Arensberg C.M., Pearsons H.W. (eds), p. 239-260

Polanyi K., Arensberg C.M., Pearson H.W. (eds) (1957), Trade and Market in the Early Empires, New York, The Free Press. 
Polanyi K., (1986), « La fallace de l'économie », Bulletin du MAUSS, n 18, p. 11-26

Polanyi K., (2002), Essais, Seuil, Paris

Polanyi M., (1962) Personal Knowledge : Toward a Post-Critical Philosophy, Chicago, Chicago University Press

Postel N., Rousseau S., Sobel R., (2006), « La responsabilité sociale et environnementale des entreprises : une reconfiguration potentielle du rapport salarial fordiste ? ", Économie appliquée, $\mathrm{n}$ ० 4 , p. 77-84

Postel N., Sobel R., (2008), « Économie et Rationalité : apports et limites de l'approche polanyienne », Cahiers d'Économie Politique, vol. 54, juin, p. 121-149

Postel N., Rousseau S., (2008), « RSE et éthique d'entreprise : la nécessité des institutions », M@n@gement, $11: 2$, p. 137-160

Posner R.A., (1973), Economic analysis of Law, little brown, Boston

Posner R.A., (2001), The economic structure of law, the collected economic essays of R. Posner, Edward Elgard

Ramaux C., (2006), Emploi : éloge de la stabilité L'État Social contre la flexicurité, Paris, Mille et une nuits

Rousseau S., (2006), « Mondialisation et concurrence des rapports à l'environnement », mondialisation : enjeux, périls et alternatives (sous la direction de Claude Serfati), éditions P.I.E-Peter Lang, Bruxelles

Rousseau S., Zuindeau B., (2007), « Théorie de la régulation et développement durable », Revue de la régulation, $\mathrm{n}^{\circ} 1$, Varia, http://regulation.revues.org, mis en ligne le 25 juin

Rubinstein M., (2006), « Le développement de la responsabilité sociale de l'entreprise : une analyse en termes d'isomorphisme institutionnel », Revue d'Économie Industrielle, $\mathrm{n}^{\circ} 113,1^{\text {ier }}$ trimestre, p. 83-105

Salais R. et Thévenot L. (eds.), (1986), Le travail - marché, règles, conventions, Paris, InseeEconomica

Salais R. et Storper M., (1994), Les mondes de production, enquête sur l'identité économique de la France, Paris, Ed. de l'EHESS

Sobel R., (Dir.), (2007), Penser la marchandisation du monde avec Karl Polanyi, Cahiers lillois d'économie et de sociologie, $\mathrm{n}^{\circ} 47$, L'Harmattan, Paris

Supiot A., (1995), Critique du droit du travail, PUF, Paris

Supiot A, (2007), « Les deux visages de la contractualisation : déconstruction du droit et renaissance féodale » in Sandrine Chassagnard-Pinet et David Hiez (dir.), Approche critique de la contractualisation, Paris, Droit et Société, L.G.D.J., p. 19-47

Thévenot L., (1986), «Les investissements de forme », in Laurent Thévenot, (ed.), Conventions économiques, Paris, Presses Universitaires de France (Cahiers de Centre d'Étude de l'Emploi), p. 21-71

Williamson O., (1985), The Economic Institution of Capitalism, New York, The Free Press 


\section{NOTES}

1. Loi $n^{\circ} 2001-420$ du 15 mai 2001 relative aux nouvelles régulations économiques.

2. Le terme de régulation hybride désigne une forme de "cogestion " de la régulation du capitalisme par les acteurs privés et publics (Peters et Pierre, 1998).

3. Outre cette définition, on peut renvoyer à l'approche classique de Bowen (1953). Sur ces éléments de définition, voir également: Ackerman, Bauer (1976) et l'excellente synthèse de Caroll (1999). Voir aussi plus récemment et en français : Capron et Quairel-Lanoizelée (2004) et Capron (2007).

4. Pour une analyse plus complète: Maucourant (2005), et sur le plan de l'analyse épistémologique : Postel et Sobel (2008).

5. «Idéologique » est ici employé au sens de représentation globale qu'un système social (ou un sous-système de ce système social) propose aux acteurs qui le font fonctionner, représentation qui donne du sens à leur actes et, dans une certaine mesure, légitime ce système et permet sa reproduction.

6. Le terme de «terre » employé par Polanyi est imprécis. Il souligne cependant: « la terre n'est que l'autre nom de la nature, qui n'est pas produite par l'homme » (Polanyi, 1983 : 107). On peut donc affirmer que ce mot de « Terre » renvoie chez Polanyi à « l'environnement naturel », c'est-à-dire aux conditions dans lesquelles la vie humaine s'est initialement développée (en dehors de tout développement économique particulier). Ce qui suppose chez Polanyi (hypothèse critiquable que nous acceptons dans le cadre de cet article) qu'il existe une "nature " indépendamment des activités humaines. On retrouve ici la thématique du caractère fini de l'environnement de l'être humain qui, confronté à ces désirs infinis, lui fait ressentir un sentiment souvent qualifié de « rareté » Dans le reste du texte nous utiliserons donc le mot « environnement naturel » en lieu et place de celui de «Terre " pour souligner le sens spécifique que Polanyi donne à ce terme.

7. Droit de propriété, respect de la concurrence, monnaie pour la mesure et les transactions (Defalvard, 1994).

8. S'agissant de la monnaie, son impossibilité à être considérée comme une vraie marchandise ne se situe pas au niveau fondamental précédent, mais à un niveau secondaire : la monnaie n'est pas une condition naturelle (comme l'est l'environnement) ou anthropologique (comme l'est le travail) de toute économie, mais la condition sociale d'une forme particulière d'économie : l'économie de marchés (c'est-à-dire en fait, le capitalisme) (Cartelier, 1996 ; Aglietta, Orléan, 1998).

9. Dans les années 1970-1980, en France notamment, différents travaux d'historiens de la pensée économique du courant «Critique de l'Economie Politique» ont entrepris de réfléchir sur les conditions de possibilité d'un paradigme hétérodoxe pouvant faire pièce au paradigme néoclassique en passe de devenir hégémonique et étant ainsi capable de produire une théorie générale des économies de marché (Cartelier, 1983; De Vroey, 1984). Leurs réflexions sur les conditions structurelles d'une marchandise (et en particulier sur le statut du travail et des ressources naturelles) sont particulièrement précieuses pour comprendre la notion de marchandise fictive chez Polanyi.

10. Dans le cas des ressources naturelles, le travail est principalement un travail d'extraction ou de conditionnement, il ne créé par la matière elle-même mais transforme cette matière en « ressource » utilisable pour le processus économique.

11. Sur la notion de raison pratique, concept aristotélicien, nous ne pouvons ici que renvoyer à l'ouvrage de Aubenque (1963).

12. Ce n'est pas ici le lieu de développer davantage la configuration historique de cette forme institutionnelle du capitalisme (le rapport monétaire et financier), mais sur le fond, il y a accord 
théorique fort entre deux macroéconomies institutionnalistes, les analyses de l'Ecole de la Régulation (Guttman, 1995) et les analyses post-keynésiennes (Lavoie, 2004 ; Van de Velde, 2005). 13. Nous usons ici à nouveau de cette expression par commodité et sans en critiquer la pertinence historique précise.

14. Le constat vaut, sur la période et pour la période de crise, pour l'ensemble des nations capitalistes avancées (Esping-Andersen, 1999).

15. Si l'on ajoute à la protection sociale, le droit du travail et les services publics, les politiques macro-économiques de type keynésien, on a les quatre piliers de ce Christophe Ramaux (2006) appelle l' « État social ».

16. C'est par exemple ce qui sous-tend le concept d'investissement de forme proposé par Thévenot (1986). D'une certaine manière la RSE relève de cette forme d'investissement dans les relations avec les parties prenantes.

17. On sait l'emprunt du concept de "personal knowledge" de Williamson au frère de Karl Polanyi, Michael Polanyi (1962).

18. Si aujourd'hui les conférences internationales n'évoquent plus de manière aussi directe les méfaits de la croissance (comme le fit le club de Rome), elles se sont en revanche centrées sur des aspects comme le réchauffement climatique et ont peu ou prou pour objectif la régulation des impacts des firmes sur le réchauffement climatique sur fond de rapports d'experts plutôt pessimistes quant au coût du réchauffement (rapport Stern, rapport du GIEC) du prix du baril qui en résulte, ou encore la faiblesse des ressources en bois, la diminution des réserves halieutiques par exemple et les autolimitations nécessaires.

19. On reconnait là les principes du courant d'analyse "law and economics", connu notamment par les travaux de Posner $(1973,2001)$ consistant à défendre une logique d'un droit subordonné à l'efficacité économique.

20. Citons par exemple, l'association Alliances ou bien encore le Forum pour l'Investissement Responsable...

21. Cet usage marchand prend une forme nouvelle avec la décolonisation et la construction d'un marché international de ressources naturelles... Qui rend immédiatement visible (par des hausses de prix et de coût d'usage) les limites de l'exploitation de la nature à des fins d'accumulation productive. Cependant, cette remachandisation ne créé pas le problème... qui est antérieur. L'exploitation de la nature et s'est poursuivi de manière continue pendant la phase de "prédation en vue de l'accumulation » qui caractérise le régime fordiste évoqué au paragraphe précédent.

22. D'une certaine manière, la RSE s'inscrit dans la perspective néo-libérale qui conçoit la régulation en amont du marché, mais pousse cette logique le plus loin possible en dénaturalisant la marchandise « terre » et la marchandise « travail ».

23. Le terme de convention renvoie bien-sûr ici aux travaux de l'économie des conventions comme nous le précisons ci dessous.

24. Déclaration de l'OIT relative aux principes et droits fondamentaux au travail adoptée en 1998.

25. Voir par exemple les travaux de Salais : Salais et Thévenot (1986), Salais et Stoper (1994).

26. Elle a en cela prolongé la redéfinition antinaturaliste de la marchandise qui sous-tend l'approche de Polanyi et que nous avons explicitée en section 1.

27. On retrouve ici les intuitions de Arrow qui indique fréquemment dans ces travaux l'idée selon laquelle: "des éléments éthiques entrent en jeu dans toute transaction, sans eux aucun marché ne pourrait fonctionner » (Arrow, $1973: 150$ ).

28. On désigne comme partie prenante: " tout groupe ou individu qui affecte ou est affecté par l'accomplissement des objectifs de l'organisation » (Freeman, (1984 : 37).

29. Il est clair que tout contrat scelle un accord sur la base d'intérêts divergents, mais ces intérêts ne reposent pas sur des positions de classe structurant le mode de production, cas dans lequel un contrat entre individu serait inopérant puisqu'il ne remettrait pas en cause les structures mêmes 
du conflit, mai sur d'inévitables divergences d'intérêt individuels. C'est en ce sens que la représentation contractualiste du règlement des conflits suppose l'absence d'un conflit social structurel inhérent au mode de production capitaliste.

30. Freeman est d'abord un philosophe moral. Il s'inspire tout à la fois de Coase (1960), pour le contractualisme, et de Rawls ou Sen pour ce qui est de la représentation « morale » de l'acteur.

31. Pour une analyse des conditions de possibilité du déploiement d'une forme de raison pratique dans l'entreprise voir Postel et Rousseau (2008) qui défendent une approche habermassienne de l'éthique.

\section{RÉSUMÉS}

Le mouvement dit de «RSE» entend concilier l'actuel mode d'accumulation à dominante financière avec certaines exigences sociales et environnementales. Or, ce mouvement s'inscrit assez bien dans l'analyse historique et institutionnaliste que Karl Polanyi a faite des limites de la marchandisation du monde. En effet, ce mouvement consiste à revenir sur la trop forte marchandisation du rapport à la nature et au travail qu'aurait entraîné le développement d'un capitalisme financiarisé. En réaction, ce mouvement mobilise l'engagement volontaire des entreprises pour bâtir une nouvelle "grande transformation", c'est-à-dire une forme inédite d'articulation entre le juste et l'efficace visant à assurer la viabilité du capitalisme. Cela revient à élargir l'engagement économique à autre chose que la simple maximisation des intérêts individuels de court terme. Cet article vise à exploiter cette proximité entre le mouvement de la RSE et l'analyse polanyienne de la marchandisation, proximité selon nous fructueuse et qui n'a jamais fait l'objet d'une analyse systématique. L'enjeu est de déterminer dans quelle mesure la RSE constitue aujourd'hui une réponse pertinente à la nécessaire gestion collective de la fiction que constitue la marchandisation apparente de la monnaie, de la terre et du travail.

The movement says about "CSR" tries to reconcile the current financial mode of accumulation with certain social and environmental requirements. This movement joins well enough in the historic and institutionnalist analysis which Karl Polanyi made of the limits of marchandisation of the world. Indeed, this movement consists in returning on excess strong marchandisation of the relationship with the nature and with the work which would have pulled the new capitalism. In reaction, this movement mobilizes the individual commitment to build a new "great transformation", that is new articulation between social justice and efficacity to assure the viability of the capitalism. It means extending economic comportment in the other thing than the simple maximization of the short-term individual interests. This article aims at exploiting this nearness between the movement of the CSR and the analysis. The stake is to determine in which measure the CSR constitutes a relevant answer to the necessary collective management of the fiction which establishes the apparent marchandisation of money, labour and nature.

\section{INDEX}

Mots-clés : marchandises fictives, Polanyi, régulation, capitalisme, responsabilité sociale des entreprises

Keywords : Fictive commodities, Polanyi, CSR, regulation, capitalism 


\section{AUTEURS}

\section{NICOLAS POSTEL}

Maître de Conférences en Économie et chercheur au Centre Lillois d'Études et de Recherches Sociologiques et Économiques (Clersé - UMR 8019 CNRS)

Bâtiment SH2 - Faculté des Sciences Économiques et Sociales de l'USTL (Lille 1)

59655 Villeneuve d'Ascq Cedex

nicolas.postel@univ-lille1.fr

\section{RICHARD SOBEL}

Maître de Conférences en Économie et chercheur au Centre Lillois d'Études et de Recherches Sociologiques et Économiques (Clersé - UMR 8019 CNRS)

Bâtiment SH2 - Faculté des Sciences Économiques et Sociales de l'USTL (Lille 1)

59655 Villeneuve d'Ascq Cedex

richard.sobel@univ-lille1.fr 